\title{
DOES USE OF ADVANCED OSTEOPERIOSTEAL FLAP RECONSTRUCT RESORBED ANTERIOR MAXILLARY ALVEOLUS BOTH VERTICALLY AND HORIZONTALLY?
}

\author{
Ahmed $\mathrm{E}^{*}$, Wael $\mathrm{E}^{* *}$ and Bahaa $\mathrm{T}^{* * *}$
}

\section{ABSTRACT}

Background: Lack of sufficient bone to place an implant at the functionally and aesthetically most appropriate position is a common problem, especially in the upper anterior jaw. Several modalities have been developed to overcome horizontal and vertical ridge atrophy in anterior maxilla with several disadvantages and limitations.

Purpose: The aim was to evaluate the reconstructed anterior maxillary alveolar ridge by using an advanced osteoperiosteal flap.

Patients and methods: Patients with atrophic edentulous anterior alveolar ridge indicated for alveolar ridge augmentation to allow rehabilitation with fixed implant-supported prosthesis were included in this study. The patients were selected from those attending outpatient clinic of Oral and Maxillofacial Surgery Department, Faculty of Dental Medicine, Al-Azhar University. Preoperative panoramic and Cone beam Computed tomography radiographs were taken for screening and measurements of both initial ridge width as well as vertical dimensions of both labial and palatal plates of bone preoperatively and immediate postoperatively.

Results: Ten partially edentulous patients were selected fulfilling the planned inclusion and exclusion criteria. Their ages ranged from 22 to 43 years with an average age of $31+/-8.06$ years old. Highly significant increase in the final ridge width. Also, noticeable coronal movement of labial plate of bone which is a part of the osteoperiosteal flap.

Conclusion: Using a labially based advanced osteoperiosteal flap allows for horizontal ridge augmentation and vertical reconstruction of labially resorbed labial plate of bone in a simple procedure.

\section{INTRODUCTION}

Atrophied premaxilla is considered one of the most problematic areas for rehabilitation with implant due to diminished ridge width and/or length. Lack of sufficient bone to place an implant at the functionally and aesthetically most appropriate position is a common problem, especially in the upper anterior jaw ${ }^{(1,2)}$. Several modalities have been developed to overcome horizontal and vertical ridge atrophy in anterior maxilla. The onlay bone grafts have been used to increase bone ridge. The ease of graft placement and immediate postoperative vertical augmentaion are the advantage of onlay grafting. However, the graft is dimensionally unstable, morbidity of second operation site and usually associated with soft tissue breakdown ${ }^{(3)}$.

It has been reported that guided bone regeneration allows for both horizontal and vertical bone

* Demonstrator of oral and maxillofacial surgery, Department of Oral and Maxillofacial Surgery, Faculty of Dental Medicine, Al Azhar University, Cairo.

** Assistant professor of oral and maxillofacial surgery, Department of Oral and Maxillofacial Surgery, Faculty of Dental Medicine, Al Azhar University, Cairo.

*** Assistant professor of oral and maxillofacial surgery, Department of Oral and Maxillofacial Surgery, Faculty of Dental Medicine, Al Azhar University, Cairo. 
augmentation in anterior maxilla but membrane exposure, graft loss and dimensional instability may occur ${ }^{(4)}$. Moreover, the distraction osteogenesis has been used for lengthening the short bone ridge but many disadvantages have been reported as distractor device instability, bone segment displacement, flap dehiscence, lack of horizontal augmentation and high cost ${ }^{(5)}$.

Nasal floor elevation combined with dental implant placement allow for placement of longer implant but not correct vertical and horizontal bone loss as the emergence profile is a must ${ }^{(6)}$. Anterior subapical osteotomy with inferior repositioning has been reported to correct vertical bone loss in anterior maxilla with the advantage of bone stability. However, it is a major procedure and still horizontal bone loss not augmented yet ${ }^{(7)}$.

The segmental osteotomy with interpositional bone grafting and simultaneous implant installation has many advantages including dimensional stability, single operation, shortened time before prosthodontic rehabilitation, and reduced gingival trauma. On the other hand, bone segment may be inclined palatally and primary stability of the implant may be questionable ${ }^{(8)}$.

In this study an intention will be paid to use a labially based advanced osteoperiosteal flap for vertical and horizontal bone augmentation in the anterior maxillary region simultaneously with the insertion of dental implants.

\section{PATIENTS AND METHODS}

Patients with alveolar bone resorption in the anterior maxilla that require alveolar ridge augmentation to allow rehabilitation with fixed implant-supported prosthesis were included in this study. The patients were selected from those attending outpatient clinics of Oral and Maxillofacial Surgery Department, Faculty of Dental Medicine, Al-Azhar University.

\section{Patient examination:}

Past and present medical history were discussed with the patients.

Full mouth examination for lesions or abnormally apparent conditions was done for all patients.

\section{Radiographic examination}

Preoperative panoramic and Cone Beam Computed tomography radiographs were taken for screening maxilla and especially anterior maxillary region.

\section{Surgical procedures}

Preoperative medication including broad spectrum antibiotic was administered intravenously one-half hour before surgery. The operation was done under local anesthesia.

Bucco-palatal incisions were composed of three incision lines; two vertical incisions opposite to the edentulous area and one horizontal incision at the ends of the two vertical incisions. This flap was extended about $6 \mathrm{~mm}$ palatally from the ridge crest. Two labial incisions were done in continuous with the two palatal vertical incisions one tooth distant from the edentulous area. Careful subperiosteal dissection was performed from the palatal incision line to crest of the ridge

The mucoperiosteum stripping was performed labially from releasing incisions till the edentulous area.

Ridge splitting had been done to separate the labial cortex from the palate one. Then, two vertical labial osteotomies (buccal cortex only) were done at the peripheries of the buccal cortical segment at least $1 \mathrm{~mm}$ from the adjacent teeth opposite to the edentulous area.

The labial plate of bone green stick out-fractured at the vestibular level cautiously by using chisels. So that, the labial plate of bone is separated from the palatal one and still attached to the labial mucoperiosteum forming the so called osteoperiosteal flap. 
The mucoperiosteum apical to the green stick out fractured labial plate of bone was stripped apically more and more. After drilling, the implant installation was preceded. Particulated cancellous

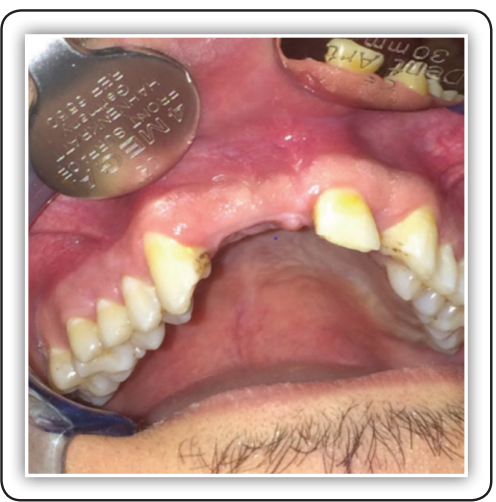

FIG (1) Showing atrophic edentulous anterior alveolar ridge missing upper right central and lateral incisor teeth with apparent healthy gingiva and good oral hygiene.

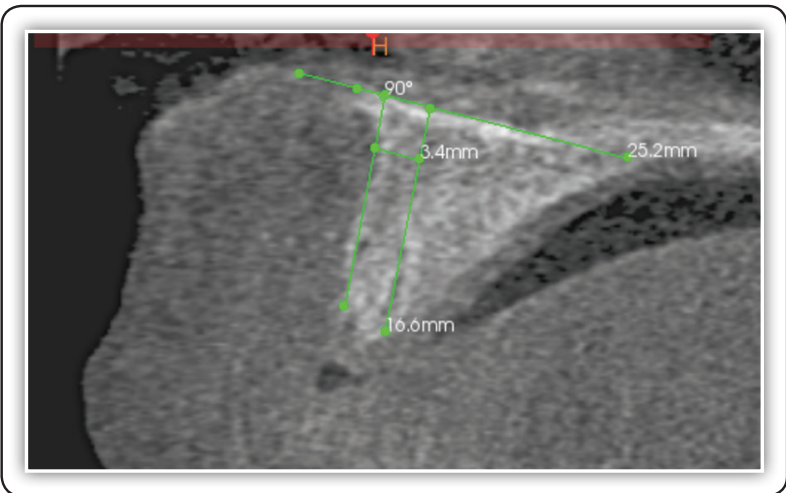

FIG (3) Showing sagittal section of CBCT at the predictable implant site showing the length of labial plate of bone extending from its crest perpendicular to a tangent to nasal floor

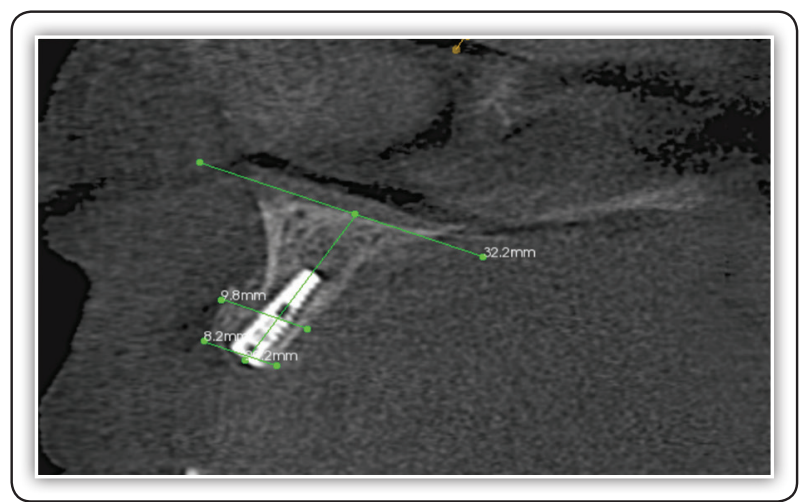

FIG (5) Showing increase in alveolar ridge width after implant installation sagittal section of CBCT. bone was harvested from the symphysis and placed around implants. Then, the osteoperiosteal flap recipient site was repositioned and sutured covering the implant fixture.

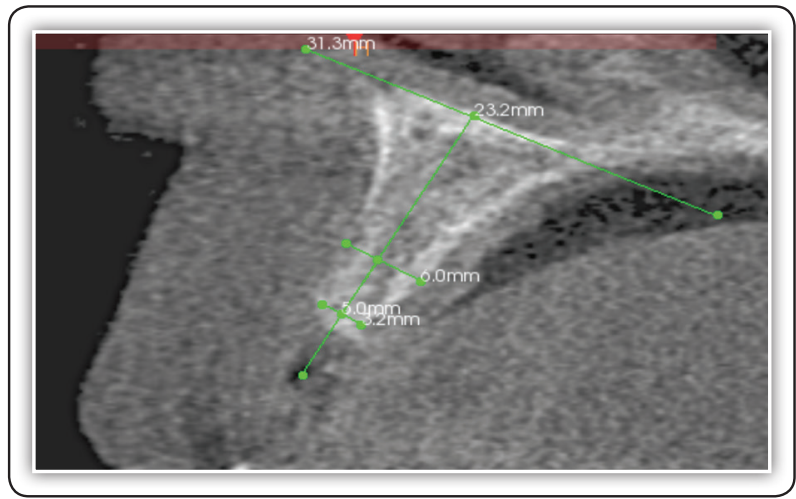

FIG (2) Showing sagittal section of CBCT at the predictable implant site showing a tangent to nasal floor was drawn. The width of the edentulous alveolar ridge was $3.2 \mathrm{~mm}$ at the crest and $6 \mathrm{~mm}$ at $5 \mathrm{~mm}$ apical to crest. Both lines were drawn parallel to the tangent of nasal floor.

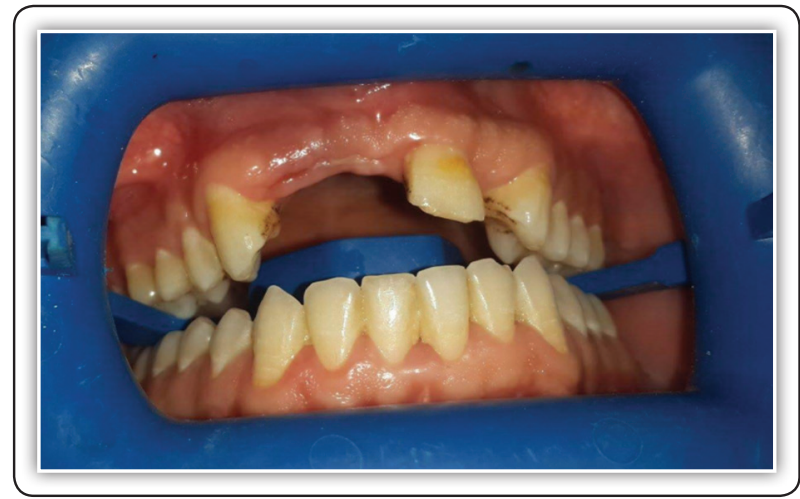

FIG (4) Showing good soft tissue healing at 2nd stage surgery

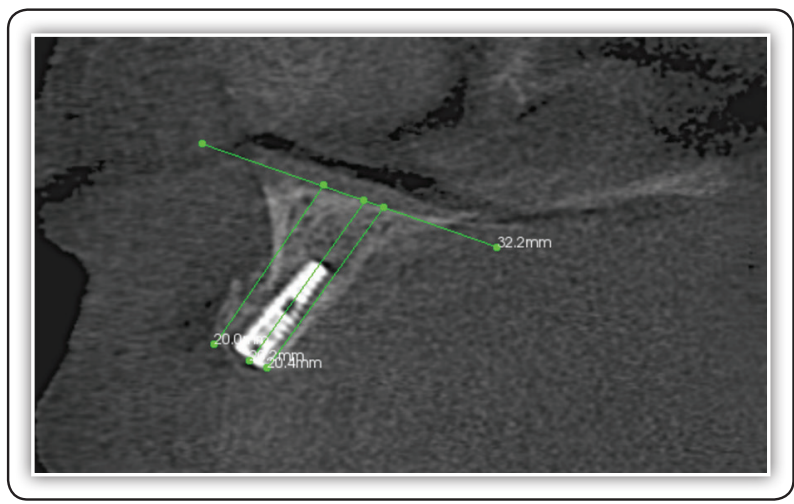

FIG (6) Showing increase in labial plate of bone height after implant placement. 


\section{Patients follow up:}

CBCT was done before surgery as well as 2 weeks after surgery to determine: initial ridge width at the crest of the ridge the length of labial plate of bone in the sagittal section from their crest to a point perpendicular to a tangent to nasal floor

\section{RESULTS}

Ten partially edentulous patients were selected from the out-patient clinic, Faculty of Oral and Dental Medicine, Al Azhar University. The selection of the patients was done fulfilling the planned inclusion and exclusion criteria. Their ages ranged from 22 to 43 years with an average age of $31+/-$ 8.06 years old.

After surgery, patients were recalled one, two, three and four weeks after implant insertion. There was mild pain at the first days that disappeared with the prescribed analgesics. There was no soft tissue dehiscence over the implant or at flap margins. The symphysial donor site was followed up clinically at the same times as the implant.

\section{Difference between initial and final ridge width.:}

The mean initial ridge width of all case was $3.74 \mathrm{~mm}$ with $\mathrm{SD} \pm 0.42$, while the mean ridge width of all cases at two weeks postoperatively was $8.18 \mathrm{~mm}$ with $\mathrm{SD} \pm 0.66$. Accordingly, the mean difference was $4.44 \mathrm{~mm}$ table (1).

TABLE (1) Showing highly statistically significant difference between initial and final ridge width

\begin{tabular}{|c|c|c|c|c|c|}
\hline \multirow{2}{*}{} & \multicolumn{3}{|c|}{ Ridge width (mm) } & \multicolumn{2}{c|}{$\begin{array}{c}\text { Paired Sample } \\
\text { t-test }\end{array}$} \\
\cline { 2 - 6 } & Mean & \pm ISD & Mean Diff. & t & p-value \\
\hline Initial & 3.74 & 0.42 & & & \\
\hline Final & 8.18 & 0.66 & 4.44 & 17.948 & $<\mathbf{0 . 0 0 1}$ \\
\hline
\end{tabular}

\section{Coronal movement of labial osteoperiosteal flap:}

Statistics analyzed the difference between preoperative and immediate postoperative amount of vertical movement of buccal flap ( $\mathrm{mm}$ ) considering the preoperative position is zero position. It showed that the mean value of vertical movement of buccal plate of bone postoperatively was $3.32 \mathrm{~mm}$ and SD was 0.89 as in table (2).

Table (2) Showing postoperative amount of coronal movement of labial plate of bone of osteoperiosteal flap which is highly statistically significant

\begin{tabular}{|c|c|c|c|c|c|}
\hline & \multicolumn{3}{|c|}{$\begin{array}{l}\text { Amount of vertical } \\
\text { movement of buccal } \\
\text { flap }(\mathrm{mm})\end{array}$} & \multicolumn{2}{|c|}{$\begin{array}{c}\text { Paired Sample } \\
\text { t-test }\end{array}$} \\
\hline & Mean & $\pm \mathrm{SD}$ & $\begin{array}{l}\text { Mean } \\
\text { Diff. }\end{array}$ & $\mathbf{t}$ & p-value \\
\hline Preoperative & 0 & 0 & \multirow[b]{2}{*}{3.32} & \multirow[b]{2}{*}{11.796} & \multirow{2}{*}{$\begin{array}{c}<0.001 \\
\text { (HS) }\end{array}$} \\
\hline $\begin{array}{c}\text { Immediate } \\
\text { Postoperative }\end{array}$ & 3.32 & 0.89 & & & \\
\hline
\end{tabular}

\section{DISCUSSION}

It is well known that implant surgery in the edentulously atrophic premaxilla is often challenging because of esthetic, phonetic, and biomechanical considerations. Ideal implant placement in prosthodontically driven location is the golden key to resolve these challenges. In other words, the fabrication of successful implant supported prostheses generally can be accomplished only if the artificial teeth are placed in the same position as was occupied by the natural teeth they are replacing. This will not be accomplished unless alveolar bone is restored to its desired form, function, and vitality in what it has been termed orthoalveolar form. Soft tissue generally follows suit, and implant placement then follows, creating a functional esthetic gingivaalveolus-implant matrix ${ }^{(9)}$.

It is concluded that successful implant need not only to be osseointegrated but also to be placed in a 3-dimensionally perfect location. Moreover, implant location must have sufficient cortical and spongy bone to ensure adequate stability and maintain good blood supply ${ }^{(10)}$. Based on clinical experience, the minimum dimensions in maxilla to insert a dental implant are an alveolar ridge width of $5 \mathrm{~mm}$ and 
a bone height of $10 \mathrm{~mm}$. Also, $1.5 \mathrm{~mm}$ to $2 \mathrm{~mm}$ of bone should remain on the buccal and lingual aspects of the implant at the bone crest Therefore, alveolar bone loss will badly affect implant placement.

This study showed high statistically significant difference between preoperative and postoperative amount of vertical movement of buccal osteoperiosteal flap. This may be due to the vertical repositioning of osteoperiosteal flap after relaxing incision which was made apically. It was agreed with Jensen et al ${ }^{(11)}$ who have introduced the island osteoperiosteal flap (I-flap) as a modified alveolar split bone grafting technique used to gain width and modify the facial or buccal bone plate position.

Results of this study showed that, the mean initial ridge width of all case was $3.74 \mathrm{~mm}$ with SD 0.42, while the mean final ridge width of all cases was $8.18 \mathrm{~mm}$ with SD 0.66. So that, the mean difference between initial and final ridge width was 4.44 $\mathrm{mm}$ which represent high statistically significant difference between initial and final ridge width. This increase in ridge width was attributed to the labially repositioned osteoperiosteal flap with the implant wedging between the two cortical plates. This labially repositioned osteoperiosteal flap allowed the widening of the coronal and middle aspects of the ridge, but the apical part maintained the same initial width. This increase in width correlated well with other published studies ${ }^{(12)}$.

In this study, there was statistically significant difference of labial marginal bone loss between immediate postoperative and after $6 \mathrm{~m}$ postoperative. This may be attributed to the minimal mucoperiosteal stripping at its superior part. This minimal mucoperiosteal stripping not only allowed for ridge exposure for splitting but also prevented from flap perforation with subsequent graft infection and implant failure. Furthermore, the tension developed on the fractured-out labial plate of bone against installed implant after repositioning of the osteoperiosteal flap and suturing during the initial healing period may be a participating cause for resorption. Also, this marginal labial bone resorption may be due to the small thickness of buccal cortex that may be occurred in some cases during ridge splitting. It was coincided with Mounir et al ${ }^{(13)}$ who concluded that more bone resorption occurred in concomitant with full mucoperiostal ridge splitting cases.

\section{CONCLUSION}

Use of a labially based advanced osteoperiosteal flap allowed for horizontal ridge augmentation as well as vertical reconstruction of labially resorbed labial plate of bone in a simple procedure.

\section{REFERENCES}

1. Adell R, Lekholm U, Rockler B, Branemark P. A 15-year study of osteointegrated implants in the treatment of the edentulous jaw. Int J Oral Surg. 1981; 10:387-416.

2. Rodriguez M, Rosenstiel F: Esthetic considerations related to bone and soft tissue maintenance and development around dental implants: report of the Committee on Research in Fixed Prosthodontics of the American Academy of Fixed Prosthodontics. J Prosthet Dent. 2012; 108:259-67.

3. Toscano N, Shumaker N, Holtzclaw D. The Art of Block Grafting A Review of the Surgical Protocol for Reconstruction of Alveolar Ridge Deficiency, The Journal of Implant \& Advanced Clinical Dentistry. 2010; 2: 45-66.

4. Le B, Rohrer M, Prasad H. Screw "tent-pole" grafting technique for reconstruction of large vertical alveolar ridge defects using human mineralized allograft for implant site preparation. J Oral Maxillofac Surg 2010; 68:428-35.

5. Ettl T, Gerlach T, Schüsselbauer T, Gosau M, Reichert T, Driemel O. Bone resorption and complications in alveolar distraction osteogenesis. Clin Oral Investig 2010; 14:481-89.

6. Mazor Z, Lorean A, Mijiritsky E, Levin L. Nasal floor elevation combined with dental implant placement. Clin Implant Dent Relat Res 2012; 14:768-71.

7. Chow L, Singh B, Chiu W, Samman N. Prevalence of postoperative complications after orthognathic surgery: a 15 -year review. J Oral Maxillofac Surg. 2007; 65:984-92.

8. Jensen O. Alveolar segmental "sandwich" osteotomies for posterior edentulous mandibular sites for dental implants. J Oral Maxillofac Surg 2006; 64:471-5 
9. Araújo M, Lindhe J. Dimensional ridge alterations following tooth extraction. An experimental study in the dog. $\mathrm{J}$ Clin Periodontol 2005; 32:212-8.

10. Handelsman M. Surgical guidelines for dental implant placement. British Dental Journal. 2006; 201:139 - 52.

11. Jensen O, Mogyoros R, Zachary Owen Z, Cottam J, Alterman M, Casap N. Island Osteoperiosteal Flap for Alveolar Bone Reconstruction. J Oral Maxillofac Surg. 2010; 68:539-46.
12. Chiapasco M, Ferrini F, Casentini P, Accardi S, Zaniboni M. Dental implants placed in expanded narrow edentulous ridges with the Extension Crest device. A 1-3-year multicenter follow-up study. Clin Oral Implants Res. 2006; 17:265-72.

13. Mounir M, Beheir G, El-Beialy W. Assessment of marginal bone loss using full thickness versus partial thickness flaps for alveolar ridge splitting and immediate implant placement in the anterior maxilla. International Journal of Oral and Maxillofacial Surgery $\cdot 2014 ; 5-21$. 